\title{
Unemployment and initiation of psychotropic medication: a case-crossover study of 2348552 Norwegian employees
}

\author{
Silje L Kaspersen, ${ }^{1,2}$ Kristine Pape, $^{1}$ Solveig 0 Ose, ${ }^{2}$ David Gunnell, ${ }^{3}$ \\ Johan Håkon Bjørngaard ${ }^{1,4}$
}

- Additional material is published online only. To view please visit the journal online (http://dx.doi.org/10.1136/ oemed-2016-103578)

${ }^{1}$ Department of Public Health and General Practice, Norwegian University of Science and Technology, Trondheim, Norway ${ }^{2}$ Department of Health, SINTEF Technology \& Society, Trondheim, Norway

${ }^{3}$ School of Social and Community Medicine, University of Bristol, Bristol, UK ${ }^{4}$ Forensic Department and Research Centre Bröset, St. Olav's University Hospital, Trondheim, Norway

\section{Correspondence to}

Silje L Kaspersen, Department of Public Health and General Practice, Norwegian University of Science and Technology, P.0. Box 8905, Trondheim 7491, Norway; silje. kaspersen@ntnu.no

Received 17 January 2016 Revised 12 April 2016 Accepted 19 April 2016 Published Online First 10 May 2016

\section{ABSTRACT \\ Objectives The study investigated initiation of psychotropic medication in relation to unemployment in the months before, during and after job loss, to detect the period of greatest risk.}

Methods The Norwegian working population in 2004 ( $N=2348$ 552) was observed from 2005 to 2010 through administrative registries linked to the Norwegian Prescription Database. A case-crossover design was used to analyse within-person relative risk of incident purchases of prescribed psychotropic drugs in relation to timing of unemployment. Control periods were defined 12,24 and 36 months before the drug purchase. Supplementary analyses were performed on medication for cardiovascular disease, diabetes, obesity, thyroid disorder, pain and musculoskeletal conditions.

Results Purchases of all psychotropic drugs increased 1-3 months before job loss. Antidepressants had the highest estimate in the month before job loss (OR 2.68, $95 \%$ Cls 2.39 to 3.01), followed by hypnotics/sedatives (OR 2.21, 95\% Cl 1.97 to 2.48), anxiolytics (OR 2.18, $95 \% \mathrm{Cl} 1.91$ to 2.48 ) and antipsychotics (OR 2.09, $95 \% \mathrm{Cl} 1.76$ to 2.48 ). Rises were greatest in men. Risk of starting psychotropic medication remained raised during a spell of unemployment, but returned to closeto-baseline levels following re-employment. Drugs used to treat somatic and pain conditions showed similar trends but with weaker associations.

Conclusions Concerns about impending unemployment may influence mental health several months prior to job loss, especially around the time of notification. The clinical implications of this might be a strengthening of preventive health initiatives early in the unemployment process.

\section{INTRODUCTION}

The association between unemployment, mental illness and suicide has been well documented, both in earlier times of economic hardship ${ }^{1}$ and in the wake of the Great Recession. ${ }^{2-4}$ Further, job loss has been related to increased risk of cardiovascular disease, sleeping problems ${ }^{5}$ and overall mortality. ${ }^{6}$ However, as poor health might lead to unemployment, ${ }^{7-11}$ and unemployment might affect health, ${ }^{12-15}$ revealing causal health effects of unemployment is an ongoing challenge in this field. ${ }^{16} 17$

People who lose their jobs are usually notified weeks or months before layoff, so the onset of

\section{What this paper adds}

- Numerous studies have reported an adverse effect of unemployment on mental health, but few studies have been able to analyse the whole unemployment process, especially how mental health is affected around the time of job-loss notification, before actual unemployment begins.

- The level of precision (exact dates) and objectivity in the ascertainment of outcomes (prescribed and purchased drugs) and exposure (registered unemployment spells) adds to a literature dominated by self-reported health measures and aggregated unemployment data.

- We found that the risk of having a first purchase of prescribed psychotropic drugs was highest 1 month before unemployment, indicating that preventive health initiatives should be strengthened around the time of notification early in the unemployment process.

- Sensitivity analyses on a range of drugs for somatic and pain conditions, not studied in the previous literature, showed similar trends as psychotropics, but with weaker associations.

possible health effects may start before the date of actual unemployment. It might be relevant to distinguish between the acute effects (shock) related to the job loss; stress caused by job insecurity ${ }^{18}$ and an anticipation of lowered income, and the effect of actually being unemployed, with its economic and social consequences. Despite this, workers' health in the days and months before unemployment is understudied, probably because lack of detailed data makes it difficult to design informative studies. In order to prevent adverse health consequences of unemployment and come up with targeted interventions, it is clinically and politically relevant to know at what time in relation to job loss people's mental health is most likely to be affected.

In this study, we investigated how mental health is affected before, during and after an unemployment spell by using first-time purchases of prescribed psychotropic medication as a measure of increased mental distress. While previous studies in this field are mainly based on self-reported and/or 
aggregated data, we had access to individual data on more than two million Norwegian employees, including exact dates of purchased medication, unemployment, vocational rehabilitation benefits, pensions, emigration and death.

To account for possible confounding factors associated with both unemployment and mental health, study participants were used as their own controls in a case-crossover design. ${ }^{19}$ We investigated how the timing of treatment initiation with prescribed psychotropic drugs in the Norwegian working population varied with unemployment spells over a 6-year period (2005-2010).

We hypothesised that mental distress related to an upcoming and ongoing unemployment spell would increase the likelihood of initiating treatment with psychotropic drugs before and during unemployment, and then decrease when the unemployment spell ended. Furthermore, we hypothesised that the increase in the likelihood of initiating drug treatment would be more pronounced for psychotropic drugs compared with drugs used for somatic conditions and pain. To the best of our knowledge, the present study is the first to analyse trajectories of several types of psychotropic and somatic drug purchases in relation to unemployment in a whole working population with detailed longitudinal data.

\section{METHODS}

\section{Data provision}

The target population comprised all inhabitants aged 1867 years, employed and resident in Norway in 2004 $(\mathrm{N}=2348$ 552). Statistics Norway provided individual-level registry data on exact dates of unemployment, social security benefits (participation in vocational rehabilitation programmes, disability pension and old age retirement), as well as sex, age, education, emigration and death from 1992 to 2011 (retirement only until 31 December 2010). The Norwegian Prescription Database was established in 2004 and provided individual-level data (dates) of all purchased psychotropic drugs from Norwegian pharmacies from 2004 and throughout the observation period (2005-2010). The drugs were identified by WHO's Anatomical Therapeutic Chemical classification system (ATC). The registries were linked using the personal identification number unique to all Norwegian inhabitants.

\section{Design and study population}

The association between timing of unemployment and incident use of psychotropic medications was analysed using a casecrossover design. By contrast with a conventional case-control design, each individual serves as his/her own control in a casecrossover design. ${ }^{20}$ Hence, all time-invariant or slow-varying confounding (eg, by sex; past psychiatric illness; educational level) is eliminated. ${ }^{19}{ }^{21}$ However, the case-crossover design still might be susceptible to time-varying confounding. ${ }^{19}$ The design assumes constant risk of exposure; the person-time in the case period is assumed to be exchangeable with the same individual's person-time during control periods. ${ }^{19}$

From the target population of 2348552 employees, we selected case-crossover samples for each group of psychotropic drugs studied; antidepressants $(\mathrm{n}=34111)$, anxiolytics $(n=32570)$, hypnotics/sedatives $(n=26838)$ and antipsychotics $(n=12495)$. Each sample consisted of employees both exposed to at least one unemployment spell, and having a prescribed psychotropic drug (outcome) dispensed during the observation period (1 January 2005-31 December 2010). In order to capture incident medication, we excluded those who purchased a psychotropic drug in $2004(\mathrm{~N}=307$ 622).
Sixteen exposure states of unemployment were defined according to timing of the unemployment spell(s): 1-6 months before, $1,2,3$, ' 4 or more' months during, and 1-6 months after the end of an unemployment spell. Further, each individual's unemployment state was recorded on the date of the first drug purchase, henceforth called the case period. Control periods were chosen 12, 24 and 36 months before the incident drug purchase took place, and each individual's unemployment state in these control periods was recorded (see online supplementary figure S1). Regarding unemployment, we only included individuals who had

1. At least one episode of unemployment lasting for $>90$ consecutive days during the observation period. Cut-off at 90 days was chosen in order to avoid the inclusion of students searching for work in holidays, and those who were registered as unemployed but secured a new job within a short time.

2. A first episode of unemployment ending no earlier than 180 days before 1 January 2005, or an unemployment spell starting within the 180 days after 31 December 2010-enabling everyone to be situated in any of the 16 time-states in the unemployment process.

See sample selection flow-chart in figure 1.

\section{Outcome ascertainment}

The outcome was having a first-time registered purchase of a psychotropic drug during the observation period (2005-2010). Separate analyses on each of the four psychotropic drug (ATC) groups were performed: N06A Antidepressants; N05C Hypnotics and sedatives; N05B Anxiolytics and N05A Antipsychotics. A list specifying the drugs and defined daily dose/1000 inhabitants/day in each group is given in the online supplementary table S1. Right censoring was done at date of death, emigration, retirement (early/old age) or long-term work disability (vocational rehabilitation programme participation, or any type of disability pension), whichever occurred first.

\section{Exposure to unemployment}

The date of unemployment was defined when an employee was registered completely out of income-producing work and signed up as $100 \%$ actively job seeking for $>90$ days, or full-time participating in re-employment programmes. The observation period was split in 30 day-intervals with a maximum of 73 periods (also referred to as months). For each of these periods, we generated dichotomous variables indicating unemployment state and first-time drug purchase based on exact dates. The dichotomous unemployment variables identified episodes of ongoing unemployment as well as the 6-month period leading up to and following each unemployment spell.

\section{Main analysis}

We compared the odds of being close to an unemployment spell in the case period (initiation of psychotropic drug treatment) with the odds of being in that same unemployment state 12, 24 and 36 months before the drug purchase took place (control periods). This takes into account seasonal variations in exposure trends, as the same months in each year are used as control periods. An indirect measure of relative risk was estimated with the OR and 95\% CIs, using a conditional logistic regression (fixed effect) estimator when comparing the odds of exposure within each individual's case and control periods. Statistical software: Stata/MP V.13. 


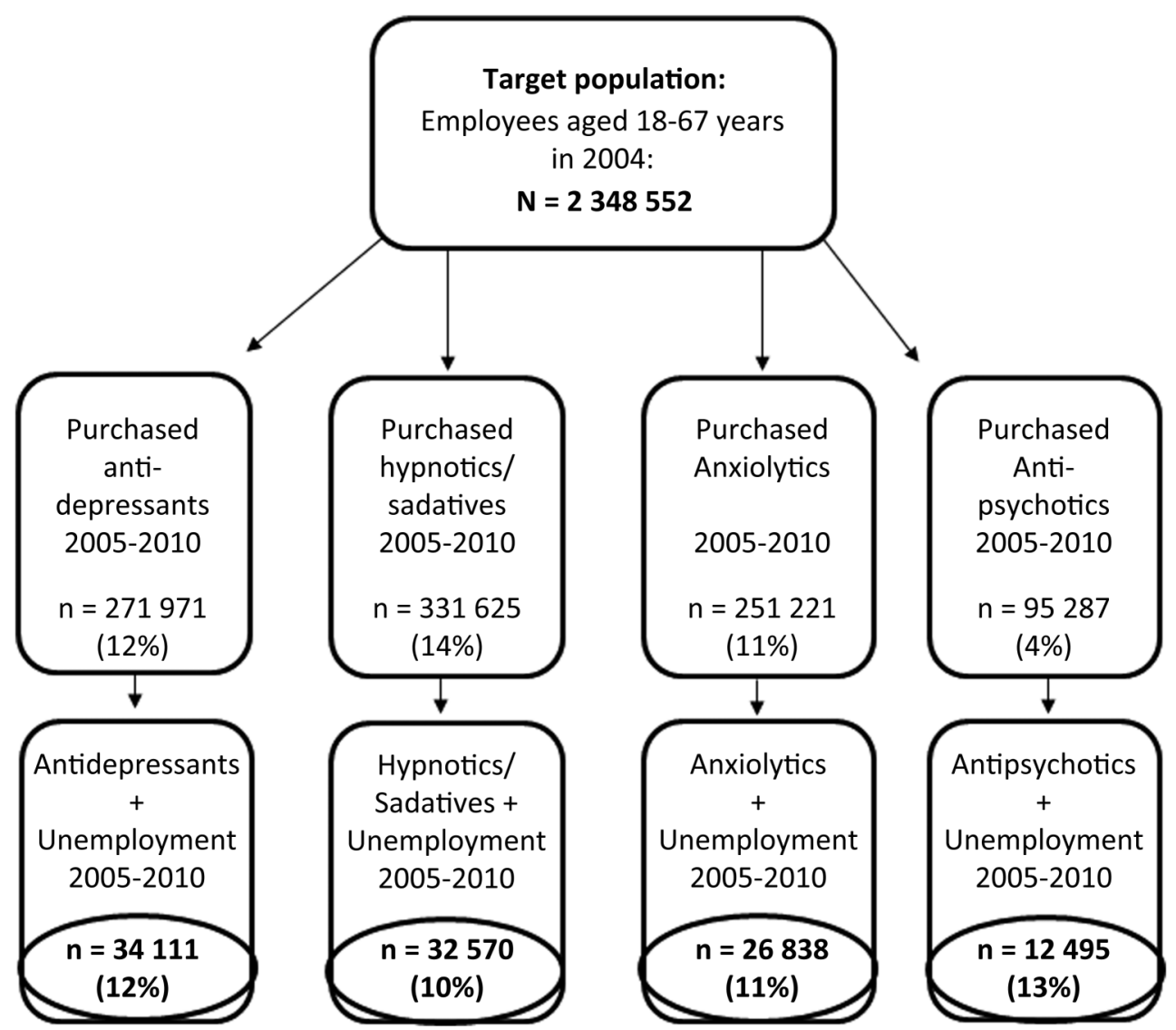

Figure 1 Flowchart of study population within each ATC group. Those with at least one purchase of prescribed psychotropic drugs (outcome) and one or more unemployment spells lasting for $>90$ days (exposure) were included in the case-crossover samples (those within the oval lines). ATC, Anatomical Therapeutic Chemical classification system.

\section{Subgroup analyses}

Subgroup (stratified) analyses were performed according to sex, age and educational level to investigate whether associations differed in these groups. Differences between groups were tested using a generalised Hausman specification test. ${ }^{22}$ Age was categorised into three groups: 18-29, 30-49 and 50-67 years. Educational level measured socioeconomic position, three categories were used: (1) compulsory education (primary school, lower secondary school or less); (2) intermediate education (upper secondary school and postsecondary non-tertiary education); (3) tertiary education (undergraduate, graduate and postgraduate).

\section{Supplementary analyses}

Previous literature has shown that length and repeated spells of unemployment are associated with deteriorations in health. ${ }^{23-25}$ To explore the effect of having several unemployment spells during the observation period, we compared individuals with multiple unemployment spells with those only experiencing one episode of unemployment.

As health selection to unemployment has been found in previous studies, ${ }^{7-9} 11$ an increase in the likelihood of purchasing psychotropic drugs around the time of unemployment could be attributed to such a selection process; individuals who develop depression may be more likely to lose their job. It is also possible that people who become depressed or anxious identify their jobs as a source of distress and decide to resign at a period their mental health is deteriorating. To explore this, we carried out supplementary analyses on purchases of drugs related to somatic conditions and pain, using the same working population and methods as the main analysis. We assessed outcomes defined by first-time purchases of medication for diabetes (ATC A10A), obesity (A08A), thyroid disorders (H03A) and cardiovascular disease (C01, C02, C03, C07, C08, C09, C10, whichever occurred first), as well as opioids (N02A), other analgesics/ antipyretics (N02B), non-steroid anti-inflammatory medication, and topical products for muscular pain (M01A and M02A).

We considered it less likely that purchases of medication for somatic conditions and pain would be systematically related to an unemployment spell. On the basis of previous literature, we anticipated some associations between unemployment status and purchase of cardiovascular disease medications, ${ }^{526}$ but overall, we expected lower estimates on somatic and pain-related drugs compared with psychotropic drugs.

\section{RESULTS}

Descriptive statistics at baseline (2004) are presented in table 1. Annual rates of unemployment in Norway over the study period ranged from $3.5 \%$ in 2005 , steadily decreasing to $1.7 \%$ in 2008, and increasing to $2.9 \%$ in 2010 . Of the 271971 (12\%) individuals in the working population purchasing antidepressants for the first time during the observation period, 34111 $(13 \%)$ had at least one unemployment spell during the observation period. Similarly, of the 331625 (14\%) incident purchasers of hypnotics/sedatives, $32570(10 \%)$ had a period of unemployment. There were 251221 individuals (11\%) who had bought anxiolytic drugs in the observation period, 26838 (11\%) of these experienced unemployment. Antipsychotic medication was 
Table 1 Descriptive statistics at baseline (2004) for all individuals who purchased psychotropic medication and for individuals both purchasing medication and being unemployed (study population) during the observation period (2005-2010)

\begin{tabular}{|c|c|c|c|c|c|c|c|c|}
\hline $\begin{array}{l}\text { Baseline } \\
\text { characteristics }\end{array}$ & $\begin{array}{l}\text { Antidepressants } \\
\text { (N06A) }\end{array}$ & $\begin{array}{l}\text { Incident } \\
\text { antidepressants } \\
\text { purchase and } \\
\text { unemployed }\end{array}$ & $\begin{array}{l}\text { Hypnotic/ } \\
\text { sedative } \\
\text { drugs (N05C) }\end{array}$ & $\begin{array}{l}\text { Incident } \\
\text { hypnotics/ } \\
\text { sedatives } \\
\text { purchase and } \\
\text { unemployed }\end{array}$ & $\begin{array}{l}\text { Anxiolytic } \\
\text { drugs (N05B) }\end{array}$ & $\begin{array}{l}\text { Incident } \\
\text { anxiolytics } \\
\text { purchase and } \\
\text { unemployed }\end{array}$ & $\begin{array}{l}\text { Antipsychotic } \\
\text { drugs (N05A) }\end{array}$ & $\begin{array}{l}\text { Incident } \\
\text { antipsychotics } \\
\text { purchase and } \\
\text { unemployed }\end{array}$ \\
\hline $\mathrm{N}$ & 271971 & 34111 & 331625 & 32570 & 251221 & 26838 & 95287 & 12495 \\
\hline Women (\%) & $159979(59)$ & $16963(52)$ & $193502(58)$ & 16068 (49) & $149828(60)$ & $14086(52)$ & $49993(52)$ & $5507(44)$ \\
\hline Age (mean/SD) & 41 (11.8) & $35(10.8)$ & $44(12.0)$ & $36(11.6)$ & $43(12.0)$ & $36(11.3)$ & $41(12.0)$ & $34(10.9)$ \\
\hline \multicolumn{9}{|c|}{ Age category (\%) (years) } \\
\hline $18-29$ & $54816(20)$ & 12659 (37) & 49845 (15) & $10679(33)$ & $41548(16)$ & $9245(35)$ & $20094(21)$ & $5045(40)$ \\
\hline $30-49$ & $145124(53)$ & 17605 (52) & 159912 (48) & $16597(51)$ & $125006(50)$ & $13737(51)$ & $49438(52)$ & $6081(49)$ \\
\hline $50-67$ & 72031 (27) & 3847 (11) & $121686(37)$ & 5294 (16) & 84667 (34) & 3856 (14) & 25755 (27) & 1369 (11) \\
\hline \multicolumn{9}{|l|}{ Education (\%) } \\
\hline Compulsory & 73815 (27) & 13354 (39) & 75846 (23) & 12253 (38) & 66774 (26) & $10920(41)$ & $27284(29)$ & $5279(42)$ \\
\hline Intermediate & $122005(45)$ & 13417 (39) & 149279 (45) & $13083(40)$ & $114902(46)$ & 10533 (39) & 42296 (44) & 4679 (38) \\
\hline Tertiary & 66287 (24) & 5049 (15) & 97444 (29) & $5312(16)$ & $62166(25)$ & 3748 (14) & 21849 (23) & 1623 (13) \\
\hline Missing (\%) & $9864(4)$ & $2291(7)$ & $9056(3)$ & $1922(6)$ & 7379 (3) & $1637(6)$ & $3858(4)$ & $914(7)$ \\
\hline $\begin{array}{l}\text { Median no days } \\
\text { unemployed; } \\
\text { (male/female) }\end{array}$ & & $392(408 / 377)$ & & $389(397 / 379)$ & & $392(406 / 384)$ & & $408(421 / 394)$ \\
\hline
\end{tabular}

Gender distribution, age (mean and SD) and proportion of individuals in each category. Median number of days of unemployment during the observation period in males (m)/females (f). No missing on gender and age.

less commonly used; 95287 (4\%) individuals purchased antipsychotics for the first time between 2005 and 2010, 12495 $(13 \%)$ of these were unemployed at some point during the observation period. Of those purchasing antidepressants, hypnotics/sedatives and anxiolytics, 52\%, 49\% and 52\%, respectively, were women, while relatively fewer (44\%) of those purchasing antipsychotic medication were women. Individuals who experienced unemployment tended to be younger than those prescribed the various psychotropic drugs as a whole.

In figure 2 we present the within-person relative risk of incident psychotropic drug purchases in relation to timing of unemployment. There was an increasing trend in psychotropic drug purchase in all medication groups 1-3 months ahead of the first registered day of unemployment, with the peak 1 month before unemployment (more than double risk), and a decrease during the unemployment spell and in particular after the end of unemployment. Of the four psychotropic drugs, antidepressants had the highest estimated ORs in the month before unemployment (OR 2.68, 95\% CI 2.39 to 3.01), followed by hypnotics/sedatives (OR 2.21, 95\% CI 1.97 to 2.48), anxiolytics (OR 2.18, 95\% CI 1.91 to 2.48), and antipsychotics (OR 2.09, $95 \%$ CI 1.76 to 2.48 ). As seen from the category ' 4 months or more' of the unemployment spell $(4+)$, there was a tendency towards increased risk of first-time psychotropic drug purchase in longer unemployment spells. Overall risk estimates of psychotropic drug purchase during all periods of unemployment (month 1-4+, data not shown) was OR 1.68 (95\% CI 1.61 to $1.76)$ in antidepressants, OR 1.47 (95\% CI 1.40 to 1.54$)$ in hypnotics/sedatives, OR 1.45 (95\% CI 1.38 to 1.53 ) in anxiolytics and OR 1.44 (95\% CI 1.34 to 1.54$)$ in antipsychotics.

\section{Subgroup analyses}

Analyses stratified by sex (figure 3) gave slightly higher risk estimates in men, especially in the months before and during unemployment. The differences in antidepressant purchases between men and women were statistically significant $(p<0.05)$ at unemployment states $-4,-3,-2,1,2$ and $4+$.
Corresponding results in hypnotics/sedatives concerned states $-5,-4,-2,1$ and $4+$; anxiolytics at states $-2,-1,1,2,4+$ and +4 ; antipsychotics at states $-5,-4,-1$ and $4+$.

Results of the age-stratified analyses (see online supplementary figure S2) showed no large differences between age groups in the months before and during unemployment. However, compared with their younger peers, the oldest employees (5067 years) seemed to have elevated risk of first-time psychotropic drug purchase also in the months after ending an unemployment spell. The analyses stratified by educational level (see online supplementary figure S3) also gave similar results as the main analysis.

\section{Supplementary analyses and robustness checks}

Of the employees included in the case-crossover samples, approximately $25 \%$ experienced more than one spell of unemployment, regardless of which psychotropic drug was studied. We performed separate analyses on employees with only one unemployment spell, and those with two or more spells during the observation period. The result (see online supplementary figure S4) showed that those experiencing only one unemployment spell, generally had higher ORs for purchasing psychotropic medication in the 3 months before and during unemployment, compared with those experiencing two or more spells.

The supplementary analyses on drugs related to more somatic conditions and symptoms are presented in figure 4. A list of medications included in each group, and descriptive statistics, are presented in the online supplementary tables S1 and S2). As expected, the associations between unemployment and first-time purchase of these were lower than that of psychotropic drug purchase. However, first-time purchases of several of these drugs showed similar patterns as psychotropic drugs in the months before unemployment. We observed increased risk of first-time purchases in the months before unemployment for antidiabetic drugs (association in the month before job loss (OR $1.44,95 \%$ CI 1.10 to 1.89 )), cardiovascular drugs (OR 1.48, 95\% CI 1.32 to 1.66 ), drugs for thyroid disorders OR 1.22, 

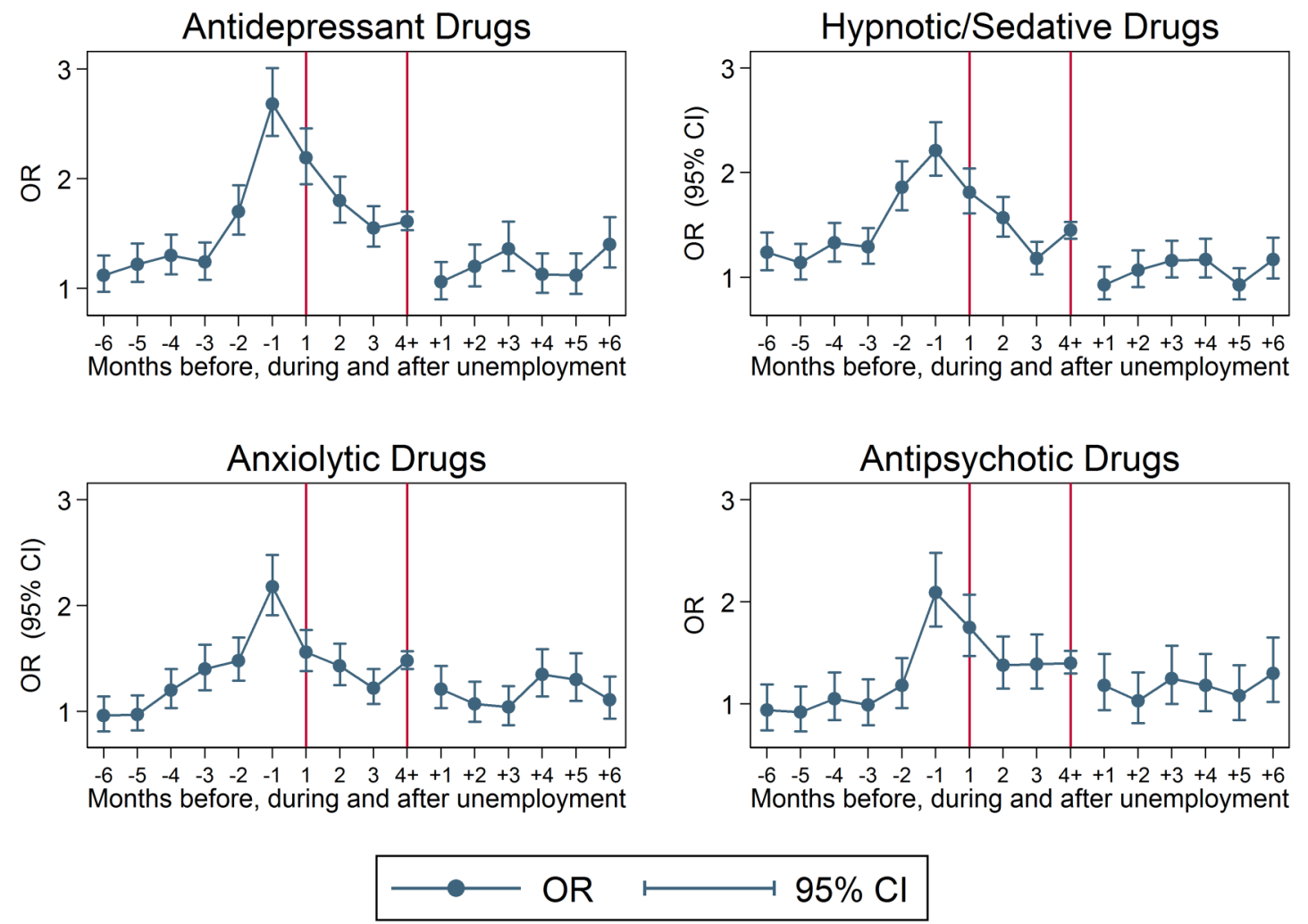

Figure 2 ORs with $95 \%$ Cls of having a first purchase of antidepressants, hypnotic/sedative drugs, anxiolytic drugs and antipsychotic drugs, respectively, while being in a state of unemployment (1-6 months before, 1, 2, 3, 4 or more months during (between vertical lines) and 1-6 months after the end of unemployment). Control periods=12, 24 and 36 months before the date of drug purchase. Start of the observation period was 1 January 2005, ending on 31 December 2010.
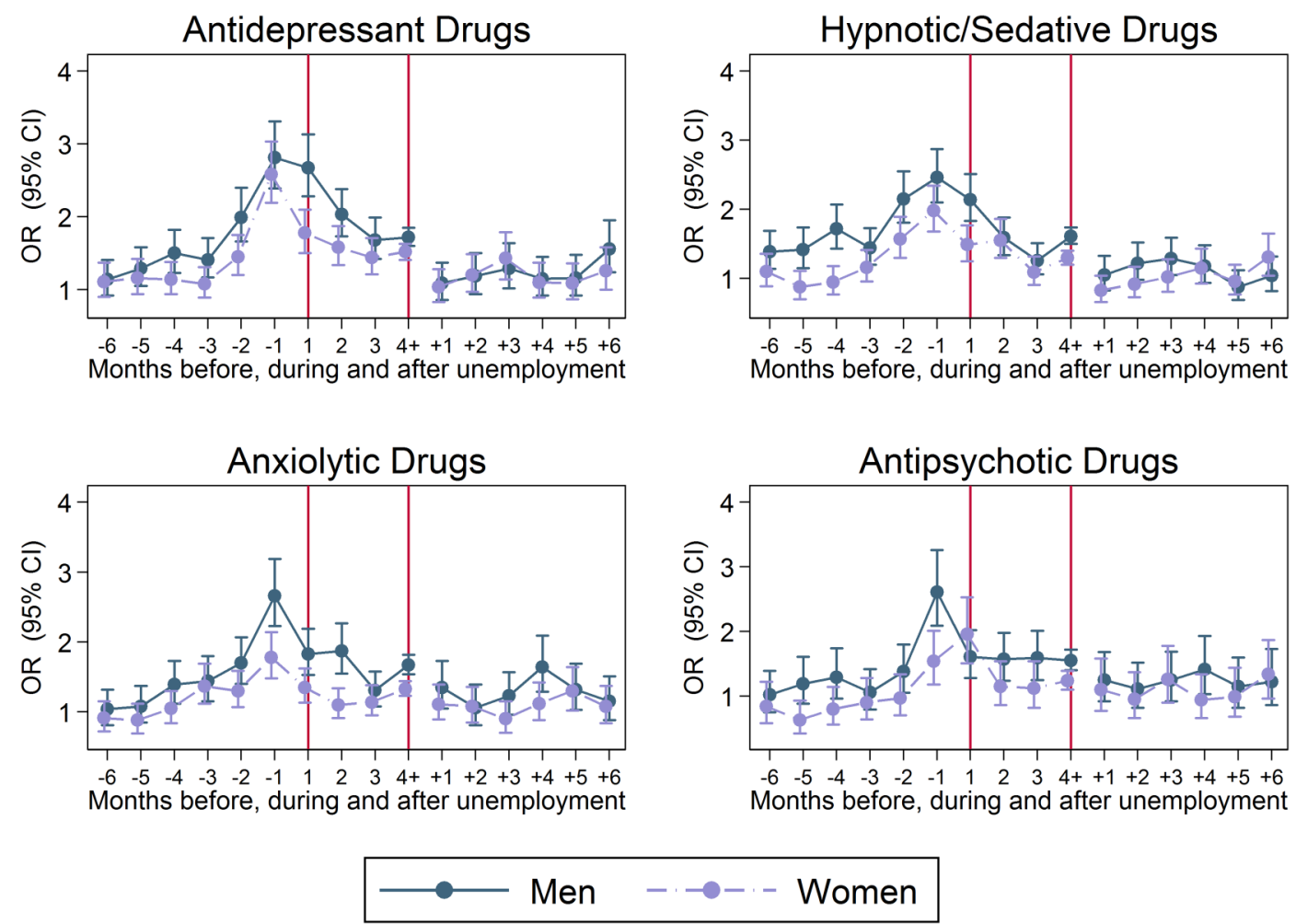

Figure 3 ORs with $95 \%$ Cls of having a first purchase of antidepressants, hypnotic/sedative drugs, anxiolytic drugs and antipsychotic drugs, respectively, while being in a state of unemployment (1-6 months before, 1, 2, 3, 4 or more months during (between vertical lines), and 1-6 months after the end of unemployment). Control periods=12, 24 and 36 months before the date of drug purchase. Start of the observation period was 1 January 2005, ending on 31 December 2010. Stratified by gender. 


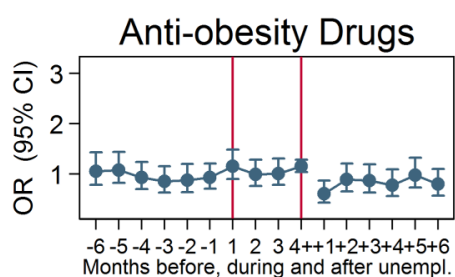

Thyroid Drugs

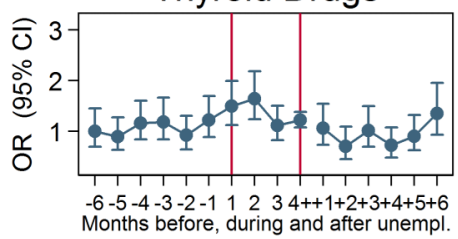

Analgesics/Antipyretics

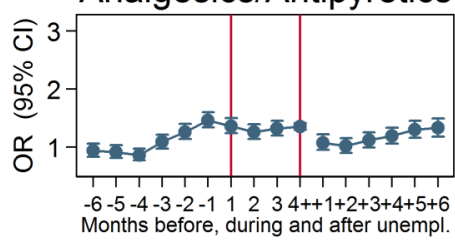

Anti-diabetic Drugs

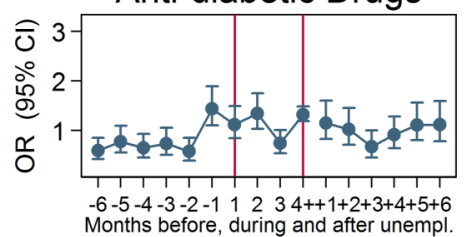

Anti-inflammatory Drugs

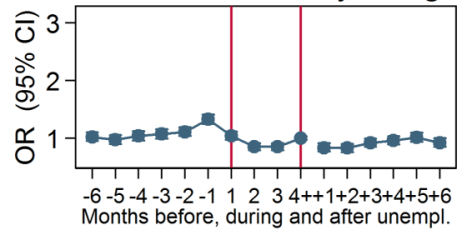

Cardiovascular Drugs
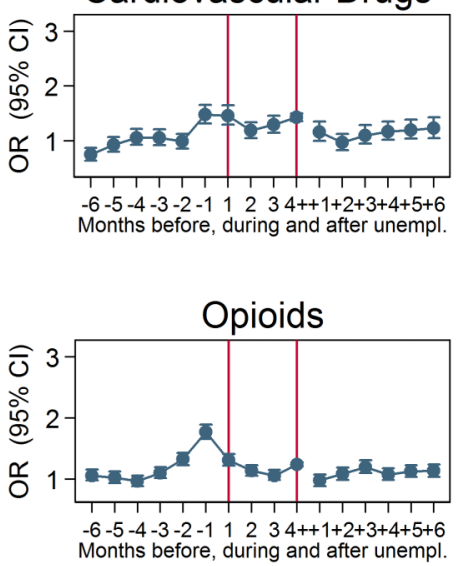

Figure 4 ORs with $95 \%$ Cls of having a first purchase of antiobesity drugs, antidiabetic drugs, cardiovascular drugs, thyroid drugs,

anti-inflammatory drugs, opioids and other analgesics and antipyretics while being in a state of unemployment (1-6 months before, 1, 2, 3, 4 or more months during (between vertical lines) and 1-6 months after the end of unemployment). Control periods=12, 24 and 36 months before the date of drug purchase. Start of the observation period was 1 January 2005, ending on 31 December 2010.

95\% CI 0.88 to 1.69 ), opioids (OR 1.77, 95\% CI 1.66 to 1.89 ) and other analgesics/antipyretic drugs (OR 1.46, 95\% CI 1.34 to 1.60). The risks of purchasing antiobesity and antiinflammatory drugs were quite similar $(\mathrm{OR} \approx 1)$ comparing case and control periods.

\section{DISCUSSION}

Analysing the initiation of psychotropic drugs before, during and after unemployment in the entire Norwegian working population from 2005 to 2010 , showed a twofold to threefold increase in the risk of first-time purchases of psychotropic drugs during the month before the date of unemployment, with an increasing trend in the 3 months ahead of unemployment. The rises were greater in men than in women. The estimated risk decreased steadily during the first 3 months of unemployment, but stayed on a higher level compared to the 6 months before unemployment. In the 6 months after the end of unemployment, the risk estimates were close to those of 6 months before job loss. Supplementary analyses on several drugs prescribed for somatic and pain conditions showed some of the same trends as psychotropic drug purchase, but with a substantially lower level of risk increase.

\section{Strengths and limitations}

A major strength of the study is the linkage of several registries, providing individual-level data on the entire Norwegian working population over a fairly long time span. The level of precision (exact dates) and objectivity in the ascertainment of outcomes (prescribed drugs) and exposure (unemployment) adds to a literature dominated by self-rated measures and aggregated data. The variety of drugs studied is also a new contribution to the literature.

Another strength is that we could assess purchases of psychotropic medications with a case-crossover design where individuals served as their own control. By design, we then eliminated all time-invariant or slow-varying confounding. Such confounding factors include sex, past psychiatric illness, educational level, genetic vulnerability and other stable individual factors relevant for the use of psychotropic drugs. However, the case-crossover design still might be susceptible to time-varying confounding, and we cannot rule out the influence from such factors in our study. Further, trends in exposure may introduce bias in casecrossover studies, ${ }^{21}$ but we consider this less likely to concern our study, as the risk of having a prescription of psychotropic drugs was equal $(\mathrm{OR} \approx 1)$ between case and control periods 6 months before unemployment.

Purchases of prescribed psychotropic drugs were used as a proxy for mental health. Prescription of psychotropic medication is based on clinical evaluation by a doctor, but is only one of several potential treatments for mental illness. Those in our study population suffering from mental illness, but not on medication, or those receiving medications while hospitalised, could not be identified in the data. Further, as we do not have available data on drug prescriptions before 2004 in Norway, 'first-time purchases' refer to the observation period and not lifetime purchases, some may have had prescriptions before 2004. This may imply that our estimates are lower than they would be if we did not have this exclusion criterion. Also, some people may have been unemployed, but for various reasons did not register as unemployed, hence not included in the study samples. 


\section{Context and generalisability}

Mental health consequences of unemployment seems to be context-sensitive, and studies from different labour markets over time are needed to add pieces to the puzzle. ${ }^{4}$ A Swedish study using monthly data on unemployment and dispensed antidepressants found no evidence of an increase in the prevalence of antidepressant use following unemployment, ${ }^{27}$ while a recent study of the Swedish working population showed an increased risk of purchasing antidepressant drugs in workers exposed to workplace downsizing. ${ }^{28}$ Evidence from the USA on macrolevel data showed that in the northeast region of the country, antidepressant and antianxiety drug prescriptions increased by $10 \%$ when employment fell by $1 \%$, while no such relationship was found in other parts of the country. ${ }^{29}$ A Dutch paper used eight waves of the European Community Household Panel $(\mathrm{N}=136556)$ to investigate how self-perceived health was affected by labour force exit due to unemployment, retirement or economic inactivity. They found a yearly increase in the likelihood of poor self-rated health after unemployment (OR 1.06, $95 \%$ CI 1.03 to 1.09), applying to all educational groups and all European regions, except the Nordic countries. ${ }^{30}$

Previous research indicates that a generous welfare state may buffer negative consequences of unemployment on mental health, measured by suicide rate and other health outcomes. ${ }^{4} 31$ This may affect the generalisability of our results outside of Norway and Scandinavia, as the social spending, level of social security and unemployment benefits and degree of unionisation is high. Also, unemployment rates in Norway have been low (below 4\%) compared with most countries in Europe during the entire observation period (including the financial crisis). Martikainen $e a^{32}$ (2006) confirmed previous findings indicating that excess mortality of the unemployed tends to be lower in regions of high unemployment, suggesting a higher degree of health selection effects when unemployment rates are low. This may concern our findings and their generalisability.

\section{Interpretation and previous findings}

The case-crossover design does not rule out the possible influence of time-varying ill health as a possible cause of job loss (ie, selection to unemployment). Still, given the detailed information on time, and incident measures of both outcome and exposure, we interpret the steadily increasing trend with a peak 1 month ahead of unemployment, as stress related to the unemployment process having a causal effect on workers' mental health. The high ORs 3-1 month before unemployment perfectly correlate in time with the general Norwegian notice period, corresponding with a plausible onset of job insecurity. Job insecurity has been found to affect the work environment and employees' mental health negatively, ${ }^{18}{ }^{28}$ in the short ${ }^{33}$ and long runs, and downsizings are often lengthy processes not only limited to the notice period. ${ }^{34}$

Being in a state of actual unemployment also implied higher risk of first-time psychotropic drug purchase compared to the risk 6 months before or 6 months after the end of unemployment. This corresponds to the previous literature on effects of unemployment on mental health referred to in the introduction. The findings that associations were stronger in men than in women are in keeping with international studies of suicides during the Great Recession, ${ }^{2}$ and studies on unemployment and health from Eastern Europe and Spain, ${ }^{12}$ while several Swedish studies found no gender differences in the unemployment effect on mental health. ${ }^{12} 35{ }^{36}$ A systematic review recently concluded that results of subgroup analyses (gender, age, educational level, marital status, etc) in this field are mixed and context dependent. ${ }^{12}$

When stratifying on one versus two or more unemployment spells, those experiencing their first and only spell had higher risk estimates than those experiencing multiple unemployment spells. A possible interpretation of this could be that the first time is the most stressful. Further, those with several spells might be employed in more unstable jobs, and have lower expectations regarding stable employment, and that unemployment did not affect their mental health as badly as in those experiencing only one spell.

The literature on unemployment effects on physical health measures (except suicide) is scarce with mixed findings. There are few relevant studies on other outcomes than cardiovascular disease. ${ }^{5}{ }^{26}$ A Swedish study did not find any effects of job loss on severe cardiovascular diseases, ${ }^{37}$ while a French study claimed that unemployment may impair cardiovascular health. ${ }^{38}$ Empirical evidence from Finland found only modest effects on mortality of unemployment due to downsizing. ${ }^{32}$ In our study, incident purchases of drugs other than psychotropics increased in the months ahead of unemployment. A plausible explanation could be an increase in morbidity (eg, pain and cardiovascular symptoms) as a response to stress and depression related to unemployment. Another explanation could be that mental distress in relation to job loss increases doctor visits per se. A doctor's visit will, in itself, trigger medical examinations likely to detect other medical problems in need of treatment. This detection aspect could contribute to an increased observed morbidity among the unemployed, previously discussed in a systematic review concluding that unemployed people may be more likely than employed people to visit physicians, take medications or be admitted to general hospitals. ${ }^{39}$ The increasing drug purchases may support the health selection hypothesis of vulnerable employees being more prone to lose their job. ${ }^{7}$ Nevertheless, the rapidly increasing ORs close to the unemployment period points towards unemployment triggering ill health.

One could expect the relationship between personal finances (which may vary over time within individuals) and medication prices to affect the demand for drugs, especially when the household economy is under pressure during unemployment. However, the Norwegian reimbursement system, to a large extent, covers the expenses for psychotropic drugs and other drugs related to chronic conditions like diabetes, thyroid disease and cardiovascular disease. In countries with high prices on medication, personal finances and market forces could confound the association between drug purchase and unemployment, but we do not consider this to be a problem in our study.

Interventions for the unemployed targeting mental health are sparsely studied. A systematic review from 2010 concluded that the evidence supporting the use of vocational interventions to improve re-employment and reduce mental distress were weak. ${ }^{40}$ However, evidence from the USA ${ }^{41}$ and Finland ${ }^{42}$ indicate that psychological interventions targeting the unemployed effectively improved mental health, and were positively associated with re-employment. A twofold to threefold risk increase in purchasing psychotropic drugs in the months before unemployment, compared with other periods of peoples' lives, means that the potential for conducting preventive healthcare should be high during this period-especially in plants going through major downsizing or closure. These are often lengthy processes that imply job insecurity for both those being laid off and those surviving in a downsizing firm. An implication of our findings could be a strengthening of preventive health initiatives early in the unemployment process, for example, through joint working between employees, 
employers, occupational health services, organisers of public re-employment programmes and general practitioners.

\section{CONCLUSION}

Although the detrimental effects of unemployment are widely recognised, the present study's results underscore that mental health may deteriorate in the period prior to the actual date of job loss. The clinical implications of this might be a strengthening of preventive health initiatives early in the unemployment process.

Contributors SLK designed the study protocol, administered the data collection process, wrote the statistical analysis plan/PhD protocol (in which the study was included), cleaned and analysed the data, and wrote most of the text in the paper. She is guarantor. KP and JHB contributed in designing the PhD protocol, analysed the data, and drafted and revised the paper. SOO contributed by designing the PhD protocol, gave suggestions on data purchases and drafted and revised the paper. DG gave advices in the analysis process, drafted and revised the paper. Consultants at Statistics Norway and the Norwegian Prescription Database prepared the data, including identifying the study participants in the registries and cooperated on registry linkages. We would also like to thank the three reviewers who contributed with useful comments in the review process.

Funding The study was part of the corresponding author's PhD project (project number 25052900) funded by the Liaison Committee between the Central Norway Regional Health Authority and the Norwegian University of Science and Technology (NTNU), including funding of data collection and registry linkage. All coauthors declare that the present study was performed independent of funders.

Competing interests All authors confirm that the recommendations for the Conduct, Reporting, Editing and Publication of Scholarly Work in Medical Journals (ICMJE Recommendations 2013) were followed. We declare that (1) SLK and KP have support from the Liaison Committee between the Central Norway Regional Health Authority and the Norwegian University of Science and Technology (PhD position and postdoctoral funding) for the submitted work; (2) SLK, KP, SOO, DG and JHB have no relationships with any companies that might have an interest in the submitted work in the previous 3 years; (3) their spouses, partners, or children have no financial relationships that may be relevant to the submitted work; (4) DG is a NIHR Senior Investigator; (5) SLK is an employee (researcher) at SINTEF Technology and Society, Department of Health. The authors declare no other relationships or activities that could appear to have influenced the submitted work.

Ethics approval The study was approved by the Norwegian Regional Committee for Medical Research Ethics (ref 2012/1941b).

Provenance and peer review Not commissioned; externally peer reviewed.

Data sharing statement No additional data is available due to restrictions from the Norwegian Data Protection Authority (Reference 13/00023-2/EOL).

\section{REFERENCES}

1 Stuckler D, Basu S, Suhrcke $M$, et al. The health implications of financial crisis: a review of the evidence. Ulster Med J 2009;78:142-5.

2 Chang S-S, Stuckler D, Yip P, et al. Impact of 2008 global economic crisis on suicide: time trend study in 54 countries. BMJ 2013:347:f5239.

3 Reeves A, McKee M, Gunnell D, et al. Economic shocks, resilience, and Male suicides in the Great Recession: cross-national analysis of 20 EU countries. Eur J Public Health 2015;25:404-9.

4 Norström T, Grönqvist $H$. The Great Recession, unemployment and suicide. J Epidemiol Community Health 2015;69:110-16.

5 Mattiasson I, Lindgarde F, Nilsson JA, et al. Threat of unemployment and cardiovascular risk factors: longitudinal study of quality of sleep and serum cholesterol concentrations in men threatened with redundancy. BMJ 1990;301: 461-6.

6 Sullivan D, von Wachter T. Job displacement and mortality: an analysis using administrative data. O J Econ 2009:124:1265-306.

7 Böckerman P, Ilmakunnas P. Unemployment and self-assessed health: evidence from panel data. Health Econ 2009:18:161-79.

8 Schuring M, Robroek SJ, Otten FW, et al. The effect of ill health and socioeconomic status on labor force exit and re-employment: a prospective study with ten years follow-up in the Netherlands. Scand J Work Environ Health 2013:39:134-43.

9 Butterworth P, Leach LS, Pirkis J, et al. Poor mental health influences risk and duration of unemployment: a prospective study. Soc Psychiatry Psychiatr Epidemiol 2012;47:1013-21.

10 van Rijn RM, Robroek SJ, Brouwer $S$, et al. Influence of poor health on exit from paid employment: a systematic review. Occup Environ Med 2014;71:295-301.
11 Kaspersen SL, Pape K, Vie GA, et al. Health and unemployment: 14 years of follow-up on job loss in the Norwegian HUNT Study. Eur J Public Health 2016;26:312-17.

12 Norström F, Virtanen P, Hammarström A, et al. How does unemployment affect self-assessed health? A systematic review focusing on subgroup effects. BMC Public Health 2014:14:1310.

13 Fergusson DM, McLeod GF, Horwood LJ. Unemployment and psychosocial outcomes to age 30: a fixed-effects regression analysis. Aust N Z J Psychiatry 2014:48:735-42.

14 Milner A, Page A, Lamontagne AD. Cause and effect in studies on unemployment, mental health and suicide: a meta-analytic and conceptual review. Psychol Med 2013:44:1-9.

15 Catalano R, Goldman-Mellor S, Saxton K, et al. The health effects of economic decline. Annu Rev Public Health 2011:32:431-50.

16 Fergusson DM, Horwood LJ, Woodward LJ. Unemployment and psychosocial adjustment in young adults: causation or selection? Soc Sci Med 2001:53:305-20.

17 Schmitz H. Why are the unemployed in worse health? The causal effect of unemployment on health. Labour Econ 2011;18:71-8.

18 Bamberger SG, Vinding AL, Larsen A, et al. Impact of organisational change on mental health: a systematic review. Occup Environ Med 2012;69:592-8.

19 Mittleman MA, Mostofsky E. Exchangeability in the case-crossover design. Int J Epidemiol 2014:43:1645-55.

20 Maclure M. The case-crossover design: a method for studying transient effects on the risk of acute events. Am J Epidemiol 1991;133:144-53.

21 Greenland S. Confounding and exposure trends in case-crossover and case-time-control designs. Epidemiology 1996;7:231-9.

22 Hausman J. Specification tests in econometrics. Econometrica 1978;46:1251-72.

23 Garcy $A M$, Vågerö $D$. The length of unemployment predicts mortality, differently in men and women, and by cause of death: a six year mortality follow-up of the Swedish 1992-1996 recession. Soc Sci Med 2012;74:1911-20.

24 Janlert $\mathrm{U}$, Winefield $\mathrm{AH}$, Hammarström $\mathrm{A}$. Length of unemployment and health-related outcomes: a life-course analysis. Eur J Public Health 2015;25:662-7.

25 Booker CL, Sacker A. Psychological well-being and reactions to multiple unemployment events: adaptation or sensitisation? J Epidemiol Community Health 2012;66:832-8

26 Virtanen M, Nyberg ST, Batty GD, et al. Perceived job insecurity as a risk factor for incident coronary heart disease: systematic review and meta-analysis. BMJ 2013;347:f4746.

27 Lundin A, Hansson A. Unemployment and dispensed prescribed antidepressants in Stockholm County 1998-09. Eur J Public Health 2014:24:666-8.

28 Magnusson Hanson LL, Westerlund $\mathrm{H}$, Chungkham HS, et al. Purchases of prescription antidepressants in the Swedish population in relation to major workplace downsizing. Epidemiology 2016;27:257-64

29 Bradford WD, Lastrapes WD. A prescription for unemployment? Recessions and the demand for mental health drugs. Health Econ 2014;23:1301-25.

30 Schuring $\mathrm{M}$, Robroek SJ, Lingsma HF, et al. Educational differences in trajectories of self-rated health before, during, and after entering or leaving paid employment in the European workforce. Scand J Work Environ Health 2015;41:441-50.

31 Cylus J, Glymour MM, Avendano M. Health effects of unemployment benefit program generosity. Am J Public Health 2015;105:317-23.

32 Martikainen $P$, Mäki N, Jäntti $M$. The effects of unemployment on mortality following workplace downsizing and workplace closure: a register-based follow-up study of Finnish men and women during economic boom and recession. Am J Epidemiol 2007;165:1070-5.

33 Østhus S. Health effects of downsizing survival and job loss in Norway. Soc Sci Med 2012:75:946-53.

34 Eliason M, Storrie D. Lasting or latent scars? Swedish evidence on the long-term effects of job displacement. J Labour Econ 2006;24:831-56.

35 Hammarström A, Gustafsson PE, Strandh M, et al. It's no surprise! Men are not hit more than women by the health consequences of unemployment in the Northern Swedish Cohort. Scand J Public Health 2011;39:187-93.

36 Strandh M, Hammarström A, Nilsson K, et al. Unemployment, gender and mental health: the role of the gender regime. Sociol Health IIIn 2013;35:649-65.

37 Eliason M, Storrie D. Job loss is bad for your health-Swedish evidence on cause-specific hospitalization following involuntary job loss. Soc Sci Med 2009;68:1396-406

38 Meneton P, Kesse-Guyot E, Méjean C, et al. Unemployment is associated with high cardiovascular event rate and increased all-cause mortality in middle-aged socially privileged individuals. Int Arch Occup Environ Health 2015:88:707-16.

39 Jin RL, Shah CP, Svoboda TJ. The impact of unemployment on health: a review of the evidence. CMAJ 1995;153:529-40.

40 Audhoe SS, Hoving JL, Sluiter JK, et al. Vocational interventions for unemployed: effects on work participation and mental distress. A systematic review. J Occup Rehabil 2010:20:1-13.

41 Vinokur AD, Price RH, Schul Y. Impact of the JOBS intervention on unemployed workers varying in risk for depression. Am J Community Psychol 1995:23:39-74.

42 Vuori J, Silvonen J, Vinokur AD, et al. The Työhön Job Search Program in Finland: benefits for the unemployed with risk of depression or discouragement. J Occup Health Psychol 2002;7:5-19. 